\title{
PATRIMONIO HISTÓRICO EDUCATIVO Y MAGISTERIO EN MÉXICO
}

\author{
Siddharta Camargo \\ Universidad Pedagógica Nacional de México \\ siddharta.camargo@gmail.com \\ Belinda Arteaga Castillo \\ Universidad Pedagógica Nacional de México \\ belindaarteagacastillo@gmail.com
}

\begin{abstract}
RESUMEN
En el presente texto se presenta una revisión de la situación normativa y de investigación del patrimonio histórico educativo en México. Es necesario aclarar que nos circunscribimos a la normatividad que atañe al patrimonio documental. En segunda instancia se revisan algunas experiencias de rescate de patrimonio histórico educativo, particularmente patrimonio documental y las metodologías empleadas. Se concluye con la mención de la necesidad de generar políticas integrales de rescate de este tipo de patrimonio, que involucre a las comunidades educativas.
\end{abstract}

Palabras clave: Patrimonio histórico educativo, archivos históricos, comunidades educativa.

\section{EDUCATIONAL HISTORICAL HERITAGE AND TEACHERS IN MEXICO}

\begin{abstract}
In this paper, I present a review of the regulatory and research situation of the educational heritage in Mexico. In first place I review laws on documental heritage in the country. Secondly I present a review of some experiences educational heritage, particularly documentary heritage and the methodologies used to conclude with the mention of the need to develop comprehensive policies to rescue this heritage.
\end{abstract}

Keywords: educational historical heritage, historical archives, educational communities.

\section{PATRIMÔNIO HISTÓRICO-EDUCATIVO E OS PROFESSORES NO MÉXICONO}

\section{RESUMO}

Neste artigo é apresentada uma revisão da situação regulamentar e de investigação e patrimônio educativo no México. Escusado será dizer que limitar-se aos regulamentos no que respeita ao patrimônio documental. Em segundo lugar resgatar algumas experiências patrimônio Histórico-Educativo, herança particularmente documental e as metodologias utilizadas são revistos. Conclui-se com a menção da necessidade de desenvolver políticas abrangentes para resgatar este patrimônio, envolvendo comunidades educativas.

Palavras-chave: patrimônio histórico educacional, arquivo histórico, comunidades educacionais. 


\section{INTRODUCCIÓN}

En México, la protección del patrimonio histórico documental se encuentra normada por la Ley Federal de Archivos y el reglamento de la misma. La Ley Federal de Archivos fue decretada el 23 de enero del 2012, mientras que el reglamento se publicó el 13 de mayo del año 2014. Ambos documentos son el resultado de un amplio proceso de consulta con diversos sectores de la sociedad, en particular con el sector académico, prioritariamente con historiadores y con archivistas, así como con otros sectores de la sociedad civil interesados en la conservación de documentos históricos.

Este marco legal permite impulsar medidas de protección del patrimonio histórico documental en la medida en la que responsabiliza con claridad a los servidores del gobierno federal del cuidado y organización de los documentos con valor histórico que se encuentren bajo su resguardo. Además estipula que quienes se encuentran en custodia de documentos con valor histórico deben garantizar las condiciones para que este patrimonio pueda ser consultado y difundido.

Las principales limitaciones de esta legislación aparecen de inmediato a los ojos del lector: ni los funcionarios de los gobiernos estatales o municipales, ni los particulares están obligados a cumplir esta ley. Las limitaciones señaladas ponen en riesgo un enorme cúmulo de documentos que se encuentran dispersos por todo el país.

Además de lo anterior, debe observarse que la ley tampoco especifica con claridad una taxonomía de los tipos de acervos documentales, esto conlleva a que se obvie la especificidad de los documentos y los lugares en los que se resguardan, es el caso del patrimonio educativo, que si bien comparte elementos con el resto del patrimonio documental, también cuenta con algunas características propias y en cuanto a los espacios en los que se ha producido y se resguarda, es decir, espacios educativos, estos también son distintos a otros espacios como las oficinas gubernamentales, las empresas u otros tipos de instituciones.

Las escuelas tienen la función de educar, en esos espacios conviven adultos, niños y jóvenes, por tanto la conservación del patrimonio histórico que se ha producido en las escuelas no es la prioridad, en realidad no merece ni siquiera atención de quienes se encuentran en inmersos en los procesos educativos, ni por supuesto, de quienes tienen a su cargo emitir las normas para dichos procesos, es decir las autoridades.

Aunado a lo anterior debemos tomar en cuenta que la visión de la historia que se enseña en las escuelas de México impone la idea de una narrativa única, preestablecida por las 
instituciones del Estado, particularmente por el gobierno federal, lo que impide la diversidad de puntos de vista y el debate y con ello, se anula la necesidad de conservar y permitir el acceso a los miembros de las comunidades educativa al patrimonio histórico documental que se ha generado y se resguarda en las escuelas.

El resultado de esta suma de circunstancias adversas es que el patrimonio histórico educativo se encuentra en riesgo permanente de ser destruido. Además cabe señalar que las circunstancias enumeradas hasta aquí, se dan en un contexto amplio plagado de tensiones y contradicciones, por un lado actores sociales e incluso políticos que presionan y construyen acuerdos para visibilizar la necesidad de conservar el patrimonio histórico como parte de una agenda amplia que garantice el acceso a la información pública gubernamental y por otro, agentes civiles, particularmente del sector político, empresarial y de seguridad del Estado que pugnan de forma soterrada por entorpecer la transparencia y el acceso a la información, llegando incluso a promover prohibiciones explícitas o negando el derecho al acceso a la información y al patrimonio histórico. Estas auténticas batallas se relacionan con la lucha por consolidar el proceso de democratización de México, un país que carece de una tradición política democrática y que cuenta con instituciones, pero frágiles y vulnerables.

Desde nuestro punto de vista resulta imperativo que las autoridades tomen las medidas necesarias y pertinentes para conservar, ordenar, clasificar y garantizar el acceso al patrimonio histórico educativo de México, tanto para su uso de investigación especializada como para un uso educativo.

\section{ALGUNOS ANTECEDENTES}

En 2002, el poder ejecutivo federal emitió la Ley de Transparencia y Acceso a la Información Pública. En ésta se señaló que los responsables de los archivos debían "contar con conocimientos y experiencia en archivística", lo cual sin duda era un acierto de los legisladores, pese a su impericia para reconocer el estado de la formación archivística en nuestro país, pues en sentido estricto sólo existía una opción educativa de profesionalización, la Escuela Nacional de Biblioteconomía y Archivonomía (ENBA) en el Distrito Federal.

En este contexto, además de expedirse diversos lineamientos e instructivos, se organizaron una serie de foros a los que fueron convocados representantes de instituciones, así como de la sociedad civil. La intención declarada por las autoridades eran las de discutir la situación de los archivos y su problemática. Aquí es importante reconocer las acciones que 
han realizado dos organizaciones paradigmáticas en México: la Asociación Civil Apoyo al Desarrollo de Archivos y Bibliotecas de México (ADABI de México, A. C.) y la Red Nacional de Archivos de Instituciones de Educación Superior (Renaies).

La Renaies se fundó en 2000, con el objetivo de conformar una conciencia y una cultura archivísticas desde las Instituciones de Educación Superior (IES) e incidir de manera responsable en la organización y preservación de sus archivos históricos concebidos como "el conjunto orgánico resultante de la acumulación natural y original de documentos - producto de las actividades administrativas de dichas entidades -, concepción que, al mismo tiempo que coadyuva al buen funcionamiento de las instituciones, garantiza la valía de los testimonios documentales para reflejar su evolución a lo largo del tiempo" (http://www.renaies.org/index.php).

Las principales Instituciones de Educación Superior (IES) públicas del país son miembros del Renaies. Entre éstas se encuentran la Universidad Nacional Autónoma de México (UNAM), la Benemérita Universidad Autónoma de Puebla, la Universidad de Sonora, la Autónoma de Sinaloa, del Estado de México, Aguascalientes, el Instituto Politécnico Nacional (IPN), así como la Benemérita y Centenaria Escuela Normal del Estado de Durango.

\section{ALgUNAS EXPERIENCIAS DE RECATE DEL PATRIMONIO HISTÓRICO EDUCATIVO EN MÉXICO}

Las instituciones que han implementado proyectos de rescate e institucionalización de archivos históricos que resguardan el patrimonio histórico educativo, con especial énfasis en el patrimonio documental, son las Instituciones de Educación Superior. Así, como hemos podido ver se debe reconocer que en la educación superior se han implementado una serie de medidas que permiten salvaguardar los acervos documentales que se han producido en los espacios universitarios y en las escuelas normales. El primer archivo histórico que se organizó en una universidad mexicana fue el Archivo Histórico de la Universidad Autónoma de México a mediados de los años sesenta del siglo XX. Tuvieron que pasar casi cincuenta años para que las universidades estatales iniciaran la fundación de sus propios archivos históricos y no fue sino hasta iniciado el presente siglo que la Secretaría de Educación Pública inició el rescate del patrimonio histórico de las escuelas normales. 
Tal como se mencionó al principio de este documento, en 2012 fue promulgada la Ley Federal de Archivos, que regula la protección de los documentos históricos producidos en el ámbito del gobierno federal. Además esta ley, promueve que todas las instituciones y ciudadanos que resguarden por alguna razón parte del patrimonio documental de la Nación lo preserven. En la redacción y gestión para la promulgación de la Ley jugaron un importante papel el equipo directivo del Archivo General de la Nación (AGN), encabezado por su exdirectora general, Aurora Gómez Galvarriato; así como del personal de la asociación civil ADABI de México, el personal del Archivo Histórico de la UNAM, de la Secretaría de la Defensa Nacional (Sedena) y la propia Renaies fueron consultados constantemente. Como se puede apreciar, se trató de un esfuerzo colectivo e interinstitucional que tanto los legisladores como el ejecutivo supieron apreciar.

Hasta el momento no se ha iniciado ningún proceso de recuperación o valoración del patrimonio histórico documental de escuelas primarias, secundarias o de jardines de niños, salvo por contadas excepciones en las que la iniciativa de exalumnos o profesores han puesto en marcha proyectos aislados y con escasos recursos, estos esfuerzos ni siquiera han sido documentados, por lo que en este momento no contamos con datos precisos que podamos citar aquí1.

\section{ARCHIVO HISTÓRICO DE LA SECRETARÍA DE EDUCACIÓN PÚBLICA}

En 2012, el Archivo Histórico de la Secretaría de Educación Pública (SEP) quedó bajo resguardo del AGN. Este repositorio surgió en 1980 como una oficina más en el marco de la Reforma administrativa, y el material documental para integrarlo fue seleccionado sin criterios archivísticos por parte de los trabajadores, quienes por fortuna tenían una formación académica en historia; más tarde se profesionalizó su organización. Cabe agregar que antes de llegar al AGN tuvo varias sedes.

El Archivo Histórico de la SEP es de gran importancia para la historia de la educación, contiene documentos que abarcan el periodo 1867-1980. Son alrededor de un

\footnotetext{
${ }^{1}$ Gracias a comentarios en congresos de historia de la educación o investigación educativa, hemos podido conocer algunos esfuerzos en zonas de la Ciudad de México, San Luis Potosí y Chihuahua. Un ejemplo interesante en este sentido es del Archivo Histórico "José María Basagoiti Noriega" del Colegio de S.I. de Loyola Vizcaínas. El Colegio fue fundado en 1732, aunque tuvo sus antecedentes en la casa fundada por los Franciscanos en la década de 1530 para recoger a las niñas mestizas que eran abandonadas por sus padres (Argueta Guerra, 2013, p. VI). Este archivo es un ejemplo de la forma en la que es posible recuperar el patrimonio histórico educativo de una institución antigua. Lamentablemente, este ejemplo sigue excepcional en México.
} 
millón de expedientes localizados en 12 mil cajas archivadoras, las cuales suman $4.5 \mathrm{~km}$ lineales de papel. Dividido en 41 secciones, éste se encuentra organizado en cuatro fondos documentales referentes a las distintas etapas históricas de la administración de la educación en México:

- Secretaría de Estado y del Despacho de Justicia e Instrucción Pública (1867-1905).

- Secretaría de Estado y del Despacho de Instrucción Pública y Bellas Artes (19051917).

- Dirección General de Educación Primaria en el Distrito Federal (1918-1921).

- Secretaría de Educación Pública (1921-1980).

La organización [actual] obedece a los principios archivísticos de procedencia y orden original, además de Fondo, Sección y Serie; entre las secciones más consultadas se encuentran: Departamento de Misiones Culturales, Instituto de Orientación Socialista, Campaña Nacional contra el Analfabetismo, Departamento de Psicopedagogía e Higiene, Dirección General de Educación Primaria en los Estados y Territorios, entre otras (RODRÍGUEZ GARCÍA, 2010, p. 260).

Para las historiadoras de la educación, el acervo custodiado por el AGN es una materia imprescindible.

\section{ARCHIVOS DE INSTITUCIONES Y/O DEPENDENCIAS EDUCATIVAS}

Los archivos que proceden de las instituciones educativas o de dependencias orientadas a la administración de la educación tienen rasgos, funciones e historias que los distinguen de los archivos generales. En primer lugar, resguardan documentación referida al servicio educativo; en segundo lugar, no dependen de la administración pública federal y, por lo tanto, su origen, funcionamiento y misión están determinados por la instancia de procedencia.

Estos archivos no sólo pertenecen y se albergan en las instituciones educativas de origen, sino que son regulados y preservados por éstas. Su fundación, organización y preservación ha corrido por muy diversos derroteros, pero en la actualidad su importancia para la historia de la educación mexicana es ampliamente reconocida. La existencia de éstos repositorios potencia "las posibilidades educativas que representan no sólo para los historiadores, archivistas, documentalistas, diplomatistas, restauradores u otros profesionales que hacen de los documentos su objeto de estudio, sino para la educación en general, incluso 
como se ha demostrado, a nivel de estudios básicos" (ISLAS, 2003, p. 16). Baste citar los archivos de las secretarías de educación en los estados y los de las universidades, así como los archivos de las escuelas normales.

\section{LOS ARCHIVOS HISTÓRICOS DE LAS ESCUELAS NORMALES}

En la historia de la educación en México aún persisten objetos de estudio abordados de manera escasa e insuficiente, éste es el caso de la historia de la educación normal y de la formación de docentes de educación básica. Esta situación puede explicarse, al menos en parte, por la carencia de archivos históricos de las escuelas normales. En muchas de estas instituciones la documentación que habla de los espacios donde se forman innumerables generaciones de educadores se encuentra dispersa en las oficinas dedicadas a los servicios escolares, la gestión administrativa o el control de personal.

Estas condiciones inadecuadas para el tratamiento de los documentos impactan negativamente en las posibilidades de reconstruir la historia, pero también en la formación de identidades que descansan sobre lo que representa la institución a lo largo del tiempo.

El programa para la fundación de la Red de Archivos Históricos de las Escuelas Normales, desarrollado por la Dirección General de Educación Superior para Profesionales de la Educación (DGESPE) en el periodo 2008-2013, pretendió generar las condiciones que permitieran el rescate, organización, inventariado, acceso y gestión de nuevos acervos documentales.

Los resultados del programa no se deben sólo a la DGESPE como instancia federal responsable de coordinar la educación normal en México, por el contrario, descansa también en el esfuerzo sostenido de las propias escuelas formadoras de docentes, en sus estudiantes, directivos, profesores, personal administrativo y manual. El concurso desinteresado y comprometido de instituciones como el AGN, la UNAM e instancias de la sociedad civil como ADABI de México y la organización "Tres siglos, tres fiestas" del estado de Chihuahua, también fueron fundamentales en este programa.

En 2008, personal adscrito a la DGESPE inició una serie de visitas y labores de diagnóstico en diversas escuelas normales de la República mexicana con la finalidad de detectar la existencia de documentos históricos, así como su estado de conservación. El diagnóstico inicial permitió documentar la existencia de documentos escritos que podían reunir las características de documentos históricos. Más aún, se logró determinar que estos 
acervos eran voluminosos, se encontraban en general en buen estado de conservación y que, sin lugar a dudas, contenían información relevante para potenciales investigaciones históricas especializadas en el campo de la historia de la educación.

Al conocer los resultados de esta primera aproximación, la DGESPE tomó la decisión de instituir un programa orientado a lograr el rescate y conservación de esos acervos. Sus objetivos generales fueron:

1) Recuperar, organizar y preservar los acervos históricos de las escuelas normales.

2) Realizar las acciones necesarias para la fundación de los Archivos Históricos de las Escuelas Normales y su posterior organización en una red que facilite la cooperación y el intercambio.

3) Realizar las gestiones conducentes a la incorporación de los archivos históricos en la Red de Archivos Históricos Mexicanos (Rahmex) y que el AGN les confiera la cédula de registro respectiva.

4) Abrir los archivos históricos de las escuelas normales a la consulta pública para fomentar y promover la investigación histórica.

5) Generar conocimientos disciplinarios de frontera y coadyuvar a la integración de cuerpos académicos en las escuelas normales vinculados a través de redes académicas con otras instituciones formadoras de docentes y con IES nacionales e internacionales.

6) Fortalecer la identidad profesional de los docentes mediante el reconocimiento de la historia de las instituciones responsables de su formación, de los personajes que contribuyeron a forjarlas, así como de sus aportaciones al desarrollo nacional y al Sistema Educativo Mexicano (SEM).

\section{EL DIAGNÓSTICO}

Con el objeto de contar con un cúmulo de información lo más completo posible, durante 2009 fue elaborada una encuesta diagnóstico para conocer el estado en el que se encontraban los acervos históricos en aquellas escuelas normales que no habían sido visitadas por el personal de la DGESPE. Dicha encuesta se entregó a los profesores que asistieron al Primer Encuentro de la Comunidad Normalista para la Educación Histórica, efectuado en la ciudad de México durante el mes de marzo. Gracias a la información de estas encuestas, más los diagnósticos elaborados por el personal de la DGESPE, fue posible tener un panorama 
general sobre las condiciones de los documentos y los retos a los que nos enfrentaríamos al efectuar la clasificación y organización de los archivos mismos.

En este diagnóstico inicial, se generaron datos acerca de 55 escuelas normales de 22 estados de la República. Las respuestas que se recibieron por parte de los profesores permitieron vislumbrar una enorme diversidad tanto en la suerte que han sufrido los acervos documentales, como en la propia historia que puede ser escrita a partir de su consulta.

Con base en los datos arrojados por las encuestas y el trabajo de campo, se observó que los documentos se encontraban dispersos en las áreas administrativas: Dirección, Control Escolar, Archivo General y Registro de Exámenes Profesionales, por mencionar algunos. Asimismo, que el tipo de documentación en todas las normales se divide al menos en tres grandes rubros: expedientes de profesores, expedientes de alumnos y organización escolar. Los primeros contienen, entre otros, información sobre los profesores de las escuelas que incluye documentos que certifican: preparación, datos personales, condiciones de trabajo en la escuela, materias que impartieron, carga horaria, planes de trabajo y exámenes que elaboraron, entre otros documentos que, como los oficios de comisión, nombramientos y cursos de actualización, dan cuenta de los movimientos de las plazas que tuvieron. Por su parte, los expedientes de alumnos contienen actas de nacimiento, los documentos que presentaron para su ingreso como cartas de buena conducta, de "pureza ideológica" y/o constancias de pobreza; certificados de estudios, cárdex, notas de extrañamiento, constancias de servicio social, actas de examen recepcional y boletas semestrales; en algunos casos, los expedientes cuentan con la fotografía del alumno. Por último, los documentos que se refieren a la organización escolar resguardan reglamentos, horarios, actas de juntas de profesores, de procesos académicos y acuerdos internos, correspondencia oficial, inventarios, libros de gastos e informes de labores, entre otros.

A su vez, el análisis posterior in situ permitió saber que las fechas iniciales de la documentación provenían en un 50\% de finales del siglo XIX y la primera mitad del siglo $\mathrm{XX}$, y se refieren a la fundación de las escuelas. Por ejemplo, en el caso de la Escuela Normal Rural de Aguilera, en Durango, se preservaron documentos que abarcan las cuatro etapas que han marcado la historia de la escuela: Central Agrícola, Escuela Regional Campesina, Escuela Práctica de Agricultura y, finalmente, Escuela Normal Rural; en el caso de la Escuela Normal Veracruzana "Enrique C. Rébsamen", se conservan numerosos documentos que el fundador de la institución generó desde 1886. En contraste, en la Escuela Nacional de Maestros no fue 
posible encontrar documentación anterior a 1940, la que se conserva es relativa a los expedientes de profesores y alumnos.

Es necesario enfatizar que no existe en México una historia uniforme y lineal de la formación de docentes en las escuelas normales. Debe hablarse de historias, en plural, pues los ritmos de los cambios históricos, los momentos de fundación y las transformaciones de las instituciones han sido por demás diversos. Desde luego que sería deseable escribir una gran summa de la historia de las escuelas normales. Esta tarea, que se antoja monumental, no podrá ser asumida por un individuo. Sin duda, tendría que tratarse de un grupo amplio de investigadores que a nivel nacional consulte de manera sistemática los archivos de dichas escuelas y de otras instituciones de educación superior. Para darnos cuenta de lo complejo del asunto, basta con mencionar el caso de la Benemérita y Centenaria Escuela Normal del Estado de Chihuahua, de la que se han contabilizado más de 100 mil expedientes de alumnos que cursaron estudios en los casi 110 años que la institución tiene de haber sido fundada. En México existen más de 230 escuelas normales públicas, lo que da una idea aproximada de la magnitud del esfuerzo que representaría escribir una historia de esta naturaleza.

\section{EL PATRIMONIO PERTENECE A LA COMUNIDAD EDUCATIVA}

Es necesario mencionar que como parte de la metodología de rescate del patrimonio documental para constituir los archivos históricos de las escuelas normales, se trató en cada caso de involucrar a la comunidad de la institución, lo cual propició una buena recepción del proyecto, cuestión fundamental si se pretende que el Archivo Histórico forme parte de las actividades cotidianas $\mathrm{y}$, por tanto, tenga una larga y productiva vida.

La cuestión no es banal, se trata de una respuesta al debate sobre la potestad y uso del patrimonio histórico. En la versión autoritaria, los usuarios son de forma exclusiva los "expertos", es decir, una élite que produce conocimiento sin la intervención de las mayorías, esta visión y estas prácticas provocan un distanciamiento de las comunidades en cuanto al patrimonio histórico y, a la larga, ese patrimonio aislado carece de valor social, por tanto, resulta irrelevante. Como se ha señalado, en este programa resultaba fundamental sensibilizar a los estudiantes normalistas sobre la necesidad de valorar, conservar y utilizar el patrimonio histórico, esto con una intención formativa que pretende lograr que estos jóvenes en el futuro cercano sean capaces de transmitir esta mirada a sus alumnos en las escuelas de educación básica. 
No puede obviarse - aunque éste no es el espacio adecuado para ahondar en ello que también nos guió la necesidad de desmitificar la historia de las escuelas normales y proveer a las comunidades normalistas de una nueva identidad para iniciar los trabajos de recuperación de su propia historia de forma profesional. Ese camino inicia con el rescate de los archivos históricos donde se encuentran las fuentes que permitirán la historiografía profesional de la educación normal en México con mayor profundidad y amplitud de la lograda hasta el momento.

Al cierre de 2011, había ya 14 archivos organizados que resguardaban 2478 cajas y 296 metros lineales de documentación que correspondían a la suma de los metros lineales de documentación localizada según la relación siguiente, señalando las fechas límite de los documentos:

- Benemérita y Centenaria Escuela Normal Veracruzana "Enrique C. Rébsamen", Jalapa, Ver. (1886-1950): 281 cajas, $33.72 \mathrm{~m}$.

- Benemérita y Centenaria Escuela Normal de Profesores del Estado de Durango, Durango, Dgo. (1876-1978): 25 cajas, $3 \mathrm{~m}$.

- Benemérita Escuela Normal de Profesores del Estado de México, Toluca, Estado de México (1930-1970): 400 cajas, $48 \mathrm{~m}$.

- Escuela Normal "Miguel F. Martínez" Centenaria y Benemérita, Monterrey, Nuevo León (1876-1960): 172 cajas, 20 m.

- Escuela Normal "Profesor Serafín Peña”, Montemorelos, Nuevo León (1876-2000): 321 cajas, $38.52 \mathrm{~m}$.

- Escuela Normal Urbana Federal de Morelia "Profesor J. Jesús Romero Flores", Morelia, Mich. (1855-1976): 411 cajas, $49.32 \mathrm{~m}$.

- Benemérita y Centenaria Escuela Normal del Estado de San Luis Potosí, San Luis Potosí (1876-1953): 111 cajas, $13.32 \mathrm{~m}$.

- Benemérita Escuela Normal del Estado de Chihuahua "Profesor Luis Urías Belderráin", Chihuahua, Chih. (1919-1993): 113 cajas, 13.56 m.

- Benemérita Escuela Normal Urbana "Profesor Domingo Carballo Félix", La Paz, Baja California Sur (1916-1975): 198 cajas, $23.76 \mathrm{~m}$.

- Escuela Normal Rural “Justo Sierra Méndez”, Hecelchakán, Campeche (1931-1967): 161 cajas, $19.32 \mathrm{~m}$.

- Escuela Normal Rural de Zacatecas "General Matías Ramos Santos", Loreto, Zacatecas, (1934-1960): 210 cajas, $25 \mathrm{~m}$. 
- Benemérita Escuela Normal de Zacatecas "Manuel Ávila Camacho", Zacatecas, Zac. (1878-2001): 75 cajas, $9 \mathrm{~m}$.

Por último, es muy importante señalar que aunque es posible documentar la existencia de documentos históricos en numerosas escuelas normales del país, por razones económicas y políticas, derivadas de la mudanza de autoridades provocado por el cambio de gobierno en el año 2012, no ha sido posible realizar su rescate, el programa de rescate del patrimonio histórico educativo, ha dejado de existir y no parece ser una prioridad de las autoridades nacionales que regulan la vida de las escuelas normales en la actualidad.

\section{LAS ESCUELAS NORMALES: METÁFORA DEL CARACOL O EL ARTE DE RECORRER CAMINOS SIN PERDER LA MEMORIA}

Para resumir en pocas palabras el contenido de los archivos históricos de las escuelas normales tendríamos que hablar de diversidad, pero también de incertidumbre y fragilidad. Ello porque nos encontramos, en términos de documentación, con la imposibilidad de trazar un esquema único que cobijara la totalidad de los acervos generados por estas instituciones.

Si nos atenemos al principio de origen y procedencia, las escuelas normales a partir de sus fuentes nos permiten dar cuenta de instituciones que no tienen un solo origen ni una procedencia universal. Por el contrario, varias de éstas nacieron como parte de los Institutos Científicos y Literarios decimonónicos - por ejemplo las del Estado de México, Chihuahua, Morelia y San Luis Potosí -, mientras que otras surgieron como proyectos autónomos (Xalapa, Guadalajara, Campeche). Además, una sola institución tuvo varias fechas de nacimiento, pues sus puertas se abrieron y se cerraron varias veces (Nuevo León, Durango, Zacatecas). Algunas fueron desde su fundación normales lancasterianas y otras estuvieron ligadas a proyectos modernizadores y científicos como los que impulsaron Enrique C. Rébsamen e Ignacio Manuel Altamirano. Por supuesto, también existen las normales del siglo $\mathrm{XX}$ que abarcan desde las normales rurales hasta las multiculturales, pasando por las regionales experimentales, los Centros Regionales de Educación Normal (CREN) y por la ahora extinta "Normal Oral", que funcionó en la ciudad de México como parte del Instituto Federal de Capacitación del Magisterio.

Con base en esta diversidad, podemos afirmar que las fechas extremas de los archivos pueden ubicarse entre finales del siglo XIX y mediados del siglo XX, pues en el caso de las normales centenarias sus archivos inician alrededor de 1855 y concluyen en 1950, 
aproximadamente. Aunque en otros casos la documentación empieza entre 1910 y 1940 y se cierra en 1970, también hay escuelas normales como la Benemérita y Centenaria Escuela Normal del Estado de Chihuahua, institución que por la forma en que se conservó la documentación, y con fines de preservación, tiene un archivo cuyas fechas extremas son: 1900-1993.

Esta compleja trama de historias implicó un desarrollo no pocas veces azaroso que marcó el destino de las escuelas normales y su documentación. Estos establecimientos nacieron de proyectos diversos pero también inestables, los cuales implicaron que estas instituciones debieran de cambiar su sede en varias ocasiones, de tal manera que prácticamente ninguna permaneció en el primer edificio que ocupó. Algunas veces, empujadas por el viento a su favor, lograron asentarse en edificios construidos ex profeso, otras, expulsadas a raíz de conflictos internos, externos o mixtos, las normales tuvieron que partir a otras latitudes.

Escuelas trashumantes, las normales más estables, como la Escuela Nacional de Maestros de la Ciudad de México, ocupó por lo menos tres edificios, al igual que la de Xalapa; otras, en cambio, como la Benemérita Escuela Normal del Estado de Jalisco y la Escuela Normal Rural "Vasco de Quiroga" de Tiripetío, Michoacán, tuvieron entre seis y ocho ubicaciones diferentes. Cada uno de estos traslados implicó la pérdida de documentación o bien su deterioro.

Ahora bien, si habláramos de patrimonio histórico educativo en una aceptación mas amplia, tal como el concepto invita a hacer, tendríamos que reconocer que en cuanto a mobiliario y edificaciones, lo que se ha conservado es bien poco y lo que puede rescatarse también. Tal es el caso de la primera sede de la Escuela Normal Veracruzana. Edificio emblemático de la escuela moderna mexicana de finales del siglo XIX, diseñado de forma cuidadosa partiendo de los principios pedagógicos a la usanza de aquel momento y que hoy en día es utilizado como sede de una escuela primaria. El edificio además ha sido objeto de diversas adecuaciones que han obviado su valor histórico y patrimonial.

Por su parte la primera sede de la Escuela Normal de Durango ha sido transformado en el Museo regional de Durango y no guarda ninguna relación con la Escuela Normal. En situaciones parecidas se encuentran la gran mayoría de los edificios que fueron construidos a finales del siglo XIX para albergar escuelas normales. Las normales han sido despojadas de su patrimonio por los vaivenes políticos. En cuanto al mobiliario y a los instrumentos de 
enseñanza, no es posible documentar su existencia, por lo que es dable pensar que han sido destruidos en su gran mayoría.

El otro capítulo del que debe hablarse es del patrimonio bibliográfico. Las escuelas normales, por la función social de forma maestros que se les asignó desde su creación y hasta el año 2013 en el que fue promulgada una reforma constitucional que anula esta potestad, organizaron bibliotecas especializadas en educación. Dichas bibliotecas contuvieron acervos de obras teóricas, pero también de manuales de enseñanza y materiales didácticos importados de Europa o escritos por los profesores y alumnos de las normales. Hasta la fecha, no existen estudios o programas institucionales que permitan valorar la riqueza de estos acervos bibliográficos, aunque el seminario permanente de historia de la educación fundado y dirigido por Luz Elena Galván ha emprendido desde el 2015 la tarea de estudiar la producción de saberes docentes a través del estudio de los manuales escolares, revistas y otro tipo de publicaciones llevadas a cabo por maestras y maestros mexicanos, muchos de los cuales se encuentran bajo resguardo de las escuelas normales.

Finalmente se debe mencionar la existencia de ricos acervos fotográficos e iconográficos que se encuentran en las escuelas normales, estas imágenes dan cuenta del paso de los años y las formas que desde la docencia y el magisterio se adoptaron para documentar y dejar testimonio de apuestas pedagógicas y momentos diversos, extraordinarios y ordinarios en la vida de estas comunidades educativas. Estos acervos tampoco han sido objeto de rescate o estudio, al menos hasta el momento.

Por estas razones - y otras que será necesario documentar -, los acervos históricos de las escuelas normales en la mayoría de los casos se encuentran incompletos y dispersos. Aún así, regresando a los documentos escritos, fueron muchos los que se conservaron, Estos nos hablan con cierto grado de detalle de la vida cotidiana en las escuelas, de las actividades de sus profesores, directivos, estudiantes y demás actores. También sobre las formas de organización académica, los acervos bibliográficos, los usos que de ellos se hacían (gracias a los libros de registros de las bibliotecas donde se anotaron los títulos y quiénes los consultaban, entre otros datos), los inventarios de compras de mobiliario y otros elementos, como los químicos, animales disecados y aparatos científicos, dan cuenta de la construcción de gabinetes científicos donde los futuros profesores del siglo XIX y principios del XX aprendieron ciencias mediante la experimentación.

Los inventarios de rudimentos, granos, animales y maquinaria agrícola, por su parte, nos hablan de los implementos de la educación rural en las escuelas normales del siglo XX; 
los planos y diseños arquitectónicos, como el dibujo que actualmente es el frontispicio de la Benemérita Escuela Nacional de Maestros diseñado por el exiliado republicano español y maestro Antonio Ballesteros Usano, nos refieren la infraestructura material, sus implicaciones simbólicas y el empleo - y apropiación - de sus espacios.

A pesar de la gran diversidad en los ritmos históricos de las escuelas normales y la huella indeleble de las transformaciones de las regiones en las que se asentaron, cabe mencionar que las que se han podido documentar por medio del análisis de los documentos de los archivos históricos, comparten una serie de características relevantes para el desarrollo de una cultura escolar propia. La primera es que sus funciones han sido normadas por el Estado mexicano desde finales del siglo XIX y que su principal función ha sido formar profesores. Es importante señalar que su relación con el Estado mexicano ha sufrido cambios relevantes, como también ha sucedido con las maneras en que se ha concebido a los profesores y, por ende, las prescripciones para su formación. En suma, los archivos históricos de las escuelas normales que hasta hoy hemos explorado nos acercan de manera profunda a los discursos, concepciones, artefactos, espacios y los actores que explican el comportamiento de estas instituciones en su diversidad.

Asimismo, se pueden conocer aspectos de la vida cotidiana de las instituciones formadoras de docentes, los ritos, prácticas y comportamientos recurrentes; los procesos de transición, las inercias, los relatos de los propios actores en torno a su paso como miembros de las comunidades normalistas, además de los complejos vínculos entre esta historia y los discursos y prácticas de los egresados en el terreno del ejercicio docente.

Actualmente nos encontramos frente a la posibilidad de remontar las historias tradicionales de la educación normal, basadas sobre todo en leyes, reglamentos y decretos de carácter general; en los discursos del poder, como es el caso de los informes presidenciales o de los secretarios del ramo, o bien, en los planes y programas de estudio cuya aplicación nacional sólo fue efectiva a partir de 1985, cuando se canceló para la Benemérita y Centenaria Escuela Normal Veracruzana "Enrique C. Rébsamen" la posibilidad de elaborar sus propios planes y programas, como sí había sucedido antes de acuerdo con el testimonio del profesor Romeo Ramírez Jiménez.

En realidad, la existencia de estas fuentes primarias y su organización en archivos históricos constituye un parteaguas en la construcción de la historia de la formación docente, ya que estos documentos nos permiten conocer el complejo pasado/presente de estas instituciones desde el panóptico de su rica, compleja y heterogénea existencia. 


\section{PALABRAS FINALES}

En este contexto, cabe destacar que la recuperación del patrimonio histórico de la educación en México permitiría recuperar en gran medida la memoria histórica del magisterio mexicano. Los historiadores de la educación, pero también los propios maestros, encontrarían nuevas fuentes que les permitiría reconstruir no sólo el paso del tiempo en las escuelas, sino sobre todo la relación de los maestros con la sociedad mexicana.

Recuperar las experiencias que han permitido que en México se rescate, valore, organice y consulte el patrimonio histórico educativo de la educación superior y en particular de las escuelas normales, podría permitir diseñar una política que se enfocara al rescate del patrimonio de las escuelas de educación básica de México, este patrimonio forma parte de la memoria del pueblo de México, por ende su rescate resulta relevante.

\section{REFERENCIAS}

AGUIRRE LORA, María Esther (coord.). Rostros históricos de la educación. Miradas, estilos, recuerdos, México, CESU/UNAM, Fondo de Cultura Económica, 2001. 328p.

ARGUeta GUERRA, María de los Ángeles. Catálogo General del Archivo Histórico “José María Basagoiti Noriega” del Colegio de S.I. de Loyola Vizcaínas. Disponible en: <http://www.colegiovizcainas.edu.mx>. Consultado el: 29 de abril del 2016.

ARTEAGA, Belinda; CAMARGO, Siddharta. Los Archivos Históricos de las Escuelas Normales y la historia ignota de la formación de docentes en México. XI Congreso Nacional de Investigación Educativa. Memorias. México, 2011. 8p. Disponible en: <http://www.comie.org.mx/congreso/memoriaelectronica/v11/docs/area_09/1761.pdf>.

Consultado el: 15 de mayo del 2013.

CAMARGO, Siddharta. Archivos Históricos de las Escuelas Normales: nuevas oportunidades para la historia de la educación en México, México, Sociedad Mexicana de Historiadores de la Educación. Biblioteca Virtual, 2010, 12p. Disponible en: <http://www.somehide.org/2012-11-29-02-50-17/biblioteca-virtual.html>. Consultado el: 15 de mayo del 2013.

DIRECCIÓN GENERAL DE EDUCACIÓN SUPERIOR PARA PROFESIONALES DE LA EDUCACIÓN. Fundación de la Red de Archivos Históricos de las Escuelas Normales. México, SEP. Disponible en: <http://www.dgespe.sep.gob.mx/comunidades/historia/investigacion>. Consultado el 15 de mayo del 2013. 
EL ARCHIVO HISTÓRICO de la Universidad Michoacana y sus publicaciones. Disponible en: <http://www.umich.mx/univ/pública/contacto/mar98/ramon2.html>. Consultado el: $21 \mathrm{de}$ abril de 2011.

GALVÁN, Luz Elena. Los maestros y la educación pública en México. México: Centro de Investigaciones y Estudios Superiores en Antropología Social, 1985. (Colección Miguel Othón de Mendizábal, 1)

GACETA IPN, número extraordinario 911, año XLVIII, volumen 14, 31de diciembre 2011.

IPN. Acuerdo por el que se expide el reglamento del archivo histórico del Instituto Politécnico Nacional. En: Gaceta Politécnica, número extraordinario 622, 31 de enero del 2006.

ISLAS, Estela. La archivística en México. México: RENAIES/BUAP, 2003.

LEY Federal de Archivos. Disponible <www.diputados.gob.mx/LeyesBiblio/pdf/LFA.pdf>. Consultado el: 26 de mayo 2012.

MENESES MORALES, Ernesto (coord.). Tendencias educativas oficiales en México 1821 - 1911. México: Porrúa, 1983.

RODRÍGUEZ GARCÍA, Alberto. Archivo histórico de la SEP. Legajos, México, Archivo General de la Nación, n. 3, enero-marzo 2010.

SOCIEDAD MEXICANA DE HISTORIA DE LA EDUCACIÓN. Disponible en: <http://www.somehide.org/home>. Consultado el: 26 de mayo de 2012.

TORRES SEPTIÉN, Valentina. La educación privada en México 1903-1976. México: El Colegio de México, Universidad Iberoamericana, 1998.

VILLANUEVA, Gustavo. La archivística objeto e identidad. México: RENAIES/BUAP/GEP, 2000.

La archivística: una ciencia en busca de sí misma (México 1915-1945). Tesis para obtener el título de Maestro en gestión documental y administración de archivos, España, Universidad Internacional de Andalucía, 2013. 